\title{
Phase transition in the countdown problem
}

\author{
Lucas Lacasa $^{1,2, *}$ and Bartolo Luque ${ }^{1}$ \\ ${ }^{1}$ Departamento de Matemática Aplicada y Estadística, Escuela Técnica Superior de Ingenieros Aeronáuticos, \\ Universidad Politécnica de Madrid, 28040 Madrid, Spain \\ ${ }^{2}$ Department of Physics, Clarendon Laboratory, University of Oxford, Oxford OX1 3PU, United Kingdom
}

(Received 30 May 2012; published 27 July 2012)

\begin{abstract}
We present a combinatorial decision problem, inspired by the celebrated quiz show called Countdown, that involves the computation of a given target number $T$ from a set of $k$ randomly chosen integers along with a set of arithmetic operations. We find that the probability of winning the game evidences a threshold phenomenon that can be understood in the terms of an algorithmic phase transition as a function of the set size $k$. Numerical simulations show that such probability sharply transitions from zero to one at some critical value of the control parameter, hence separating the algorithm's parameter space in different phases. We also find that the system is maximally efficient close to the critical point. We derive analytical expressions that match the numerical results for finite size and permit us to extrapolate the behavior in the thermodynamic limit.
\end{abstract}

DOI: 10.1103/PhysRevE.86.010105

PACS number(s): 05.70.Fh, 89.20.Ff, 89.75.-k

In combinatorial optimization problems, a large amount of literature is directed at the occurrence of a so-called threshold phenomenon in the performance of search algorithms [1-4]: There exists a phase in parameter space where the search algorithm can easily find a solution to the aforementioned combinatorial problem (as the number of available solutions increases exponentially with the system size) and a phase where such a solution typically (i.e., almost surely) does not exist. The transition between both phases is sharp in some situations, mimicking in several aspects the phenomenon of a phase transition in statistical physics problems. Some classical problems evidencing such a phenomenon include combinatorial problems in random graphs or the satisfaction of (random) Boolean clauses, generically gathered under the umbrella of random constraint satisfaction problems [4,5]. Many of these concrete problems can indeed by interpreted under a statistical physics formalism [6,7], the general idea being that in a combinatorial optimization problem, in some cases one can formalize a cost function to be minimized. In satisfaction problems, this is, for instance, the number of violated constraints. Within statistical physics of disorders systems, such as in spin glasses, one indeed proceeds in the same manner if the system is studied in the limit of low temperature (in that situation, the system tries to adopt the ground state or minimal energy configuration). Thereby, the cost function of a combinatorial optimization problem can be related to the Hamiltonian of a disordered system at zero temperature (for instance, finding a minimum partition within the so-called partitioning problem is equivalent to finding the ground state of an infinite-range Ising spin glass with Mattis-like, antiferromagnetic couplings [7]).

In this work we present a random decision problem, called the countdown problem, which is inspired by a celebrated British television quiz show called Countdown (based on the French game show Des Chiffres et des Lettres, one of the longest-running game shows in the world, and called other names in several countries [8]). This show consists of several

\footnotetext{
*lucas.lacasa@upm.es
}

games, one of which incorporates the combinatorial problem of arithmetically combining several numbers to produce another one. Concretely, the contestants must use arithmetics to reach a given target number from six other numbers, using each of them at most once. Here we formalize a random version of this decision problem and explore its solvability as a function of the parameter space. We will provide numerical evidence according to which the solvability of the decision problem shows the presence of an algorithmic phase transition and introduce an approximate theory, which we show to be in good agreement with the numerics for finite-size systems and permits us to theoretically find the phase transition in the thermodynamic limit.

Let us begin by defining the pool of size $M$ as the integer interval $[1, M]$. Suppose that we randomly extract with reposition from this pool a set of $k$ integers $\mathcal{X}=$ $\left\{x_{1}, x_{2}, \ldots, x_{k}\right\}$ and another integer $T$ called the target. Stated as a decision problem, the target game raises the following question: For a given duple $(k, M)$, what is the probability $P(k, M)$ of reaching $T$ by combining the elements of $\mathcal{X}$ (where each element $x_{i}$ can be used or not, but each of them will be used at most once) through the set of arithmetic operations $\mathcal{A}=\{+,-, \times, \div\}$ ? Once $M$ is fixed, one can assert that for rather small values of $k$, such as $k=2$, the number of possible combinations is rather limited. For large values of $k$, the situation is the opposite: On average there will exist many possible ways of combining the elements in $\mathcal{X}$ to reach $T$. Whereas several parallels with random $k$ satisfiability can be outlined, it can be shown that the problem at hand is not based on finding the correct variables assignment $\mathcal{X}$, but the correct Hamiltonian assignment $\mathcal{H}$ among a Hamiltonian ensemble [9]. While this dual representation precludes the possibility of a standard statistical mechanics approach, we will take advantage of the number-theoretic nature of the problem to propose a probabilistic-theoretic treatment. We start be relaxing the problem statement by assuming that the set of available arithmetic operations is restricted to $\mathcal{A}=\{+,-\}$. As the problem is computationally nontrivial [10], in order to explore the problem numerically, we have implemented a brute force recursive routine that explores the search space in 


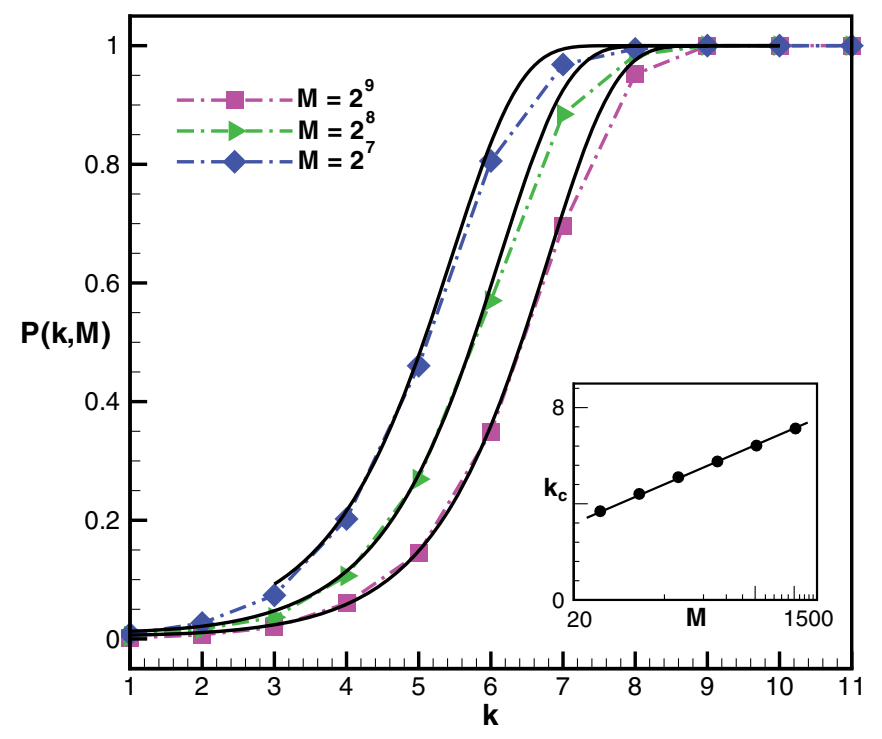

FIG. 1. (Color online) Winning probability $P(k, M)$ curve as a function of $k$, for increasing pool sizes $M$, in the system with $\mathcal{A}=\{+,-\}$. Solid curves are the analytical predictions [Eqs. (2)-(4). The inset shows a linear-logarithmic plot of the crossover value scaling $k_{c}(M)$ for different values of the pool size $M$. In each case the crossover value is estimated as the linear interpolation of $k$ for which $P\left(k_{c}, M\right)=0.5$, as in percolation theory. The straight line is a fit to a logarithmic function $k_{c}(M)=a \ln (M)+b$ with $a=0.98$ and $b=0.31$.

an exhaustive way (additional details are given in Ref. [9]). For a given pool size $M$, we fix $k$, make Monte Carlo simulations, and perform ensemble averages over different realizations of $T$ and $\mathcal{X}$. The winning probability $P(k, M)$ is defined as the probability of reaching $T$ by arithmetically combining at most the $k$ numbers (where each of the $k$ elements can be used or not, but each of them will be used at most once). In Fig. 1 we plot the results of $P(k, M)$ vs $k$ for different pool sizes $M$, averaged in each case over $10^{4}$ realizations. Note that the transition between $P \sim 0$ (losing almost surely) and $P \sim 1$ (winning almost surely) is sharp and occurs at a certain $k_{c}(M)$, estimated as the linear interpolation of $k$ for which $P\left(k_{c}, M\right)=0.5$, as usual in percolation theory. This crossover value depends on the pool size since the control parameter $k$ is not intensive. In the inset of Fig. 1 we plot, on a linear-logarithmic scale, its dependence on system size, finding a logarithmic scaling of the form $k_{c}(M)=a \ln (M)+b$, with $a=0.98$ and $b=0.31$.

Our analytical treatment deals with the estimation of $P(k, M)$, where $k \ll M$, and for that task we begin by considering a single operation, the sum. If we choose at random $n$ numbers from $[1, M]$, the largest possible value of its sum is $n M$. In a first approximation, we will suppose that the sum of $n$ numbers is uniformly distributed in $[1, n M]$. The result of this sum will fall in $[1, M]$ with probability $1 / n$. The number of results in $[1, M]$ accessible from $k$ numbers chosen at random from that interval is therefore $\sum_{n=1}^{k} \frac{1}{n}\left(\begin{array}{l}k \\ n\end{array}\right)$ (note at this point that arguments involving the central limit theorem are not employed here as long as we are dealing with partial sums of $2,3, \ldots, k$ random variables instead of simple sums of $k$ random variables and in the general case of more arithmetic operations the theorem does not apply).
Let us proceed by introducing a second operation in our system: substraction. Incorporating this operation is analogous to handling the former system, where now each number $a$ from the $n$ numbers chosen at random represents both $a$ and $-a$. Now the result of summing up the $2 n$ numbers will belong to $[-n M, n M]$, such that, assuming uniformity once again, it will fall in $[1, M]$ with probability $1 / 2 n$. Proceeding as before, the number of results in $[1, M]$ accessible from $k$ numbers chosen at random from that interval $N(k)$ can be written as

$$
N(k)=\sum_{n=1}^{2 k} \frac{1}{2 n}\left(\begin{array}{c}
2 k \\
n
\end{array}\right) \approx \frac{2^{2 k}-k-2}{2 k+1} .
$$

The third approximation consists in assuming that those $N$ by-products are indeed random independent trials of finding the target in $[1, M]$. In that situation, the winning probability $P(k, M)$ reads

$$
P(k, M)=1-\left(1-\frac{1}{M}\right)^{N(k)} \approx 1-e^{-N(k) / M}
$$

for $M \gg 1$. Although this result is based on crude approximations assuming independence and uniformity [which yields a value for $N(k)$ that is independent of $M$ ], it still works qualitatively (results not shown). In order to gain quantitative accuracy, we can take Eq. (1) up to leading order and as an ansatz we introduce a dependence on $M$ through a modulating factor $r(M)$ that quantifies the correlations among numbers (i.e., the lack of independence) such that

$$
N(k, M)=\frac{e^{r(M) k}}{k} .
$$

In order to estimate $r(M)$, note that $k_{c}(M)$ is defined such that $P\left(k_{c}, M\right)=1 / 2$. From Eq. (2) we find

$$
r(M)=\frac{\ln \left(M k_{c} \ln 2\right)}{k_{c}} \text {. }
$$

In Fig. 1 we plot, as solid lines, the theoretical values of $P(k, M)$ as a result of Eqs. (2)-(4), with the appropriate scaling $k_{c}(M)$ previously reported: The ansatz yields a selfconsistent result provided $k_{c}(M)$ is a logarithmic function. In Fig. 2 we extend the problem to the more general case in which all elementary arithmetic operations are allowed, $\mathcal{A}=\{+,-, \times, \div\}$. The numerical results are analogous to the simpler case, where now the finite-size scaling of the critical point fulfills $k_{c}(M)=a \ln M+b$, with $a=0.84$ and $b=0.39$. Solid lines are the predictions of the theory with the latter scaling, showing again good agreement with the numerical simulations.

Introducing the intensive control parameter $\alpha=k / k_{c}$, in Fig. 3 we plot the numerical curves $P(\alpha, M)$ resulting from the simulations performed in the general case. As the system size increases, the probability of satisfying the game gets sharper around the now-size-independent crossover value. The behavior in the thermodynamic limit $(M \rightarrow \infty, \alpha$ finite) can be derived from Eqs. (2)-(4), finding a Heaviside step function

$$
P_{\infty}(\alpha)=\lim _{M \rightarrow \infty} 1-e^{-e^{r(M) \alpha k_{c}} / \alpha k_{c} M}= \begin{cases}0 & \text { for } \alpha<1 \\ 1 & \text { for } \alpha>1,\end{cases}
$$

i.e., the onset of a true phase transition.

Finally, let us define the function $Q(k, M)$ that measures the system's efficiency as the average amount of potential targets 


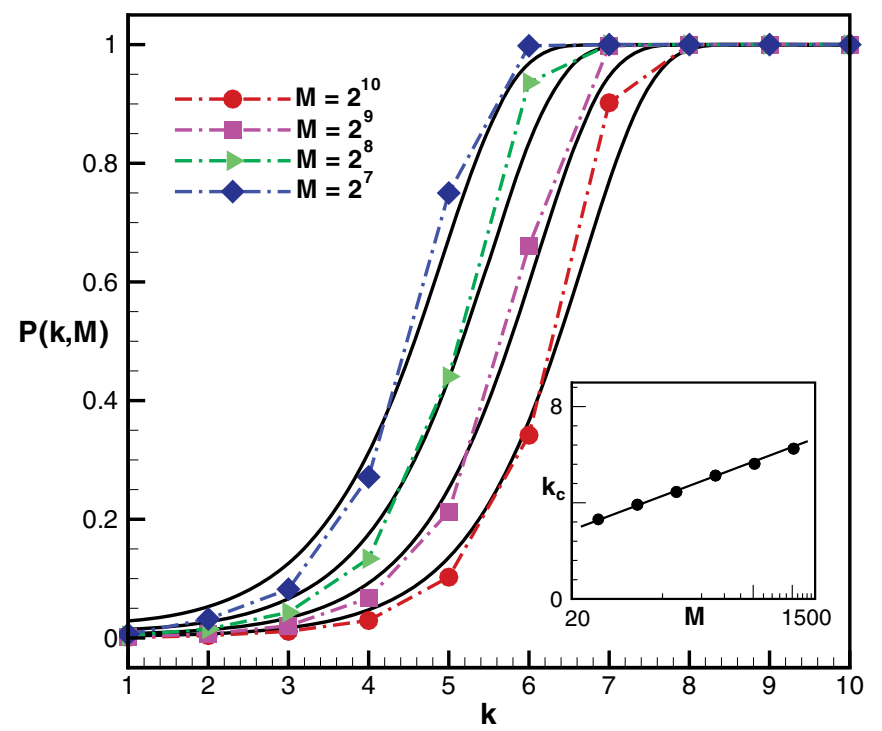

FIG. 2. (Color online) Numerical results similar to those in Fig. 1 in the general case of $\mathcal{A}=\{+,-, \times, \div\}$. The scaling of the critical point is again logarithmic $k_{c}(M)=a \ln (M)+b$, with $a=0.84$ and $b=0.39$, which shifts the critical point towards smaller values of $k$. Solid lines are the results of the theory [Eqs. (2)-(4)] with the latter scaling relation.

in $[1, M]$ that can be reached per unit number $k$. This function can be written as

$$
Q(k, M)=\frac{P(k, M) M}{k},
$$

whose behavior is shown in Fig. 4. This measure reaches a maximum in a neighborhood of $k_{c}(M)$ such that in the

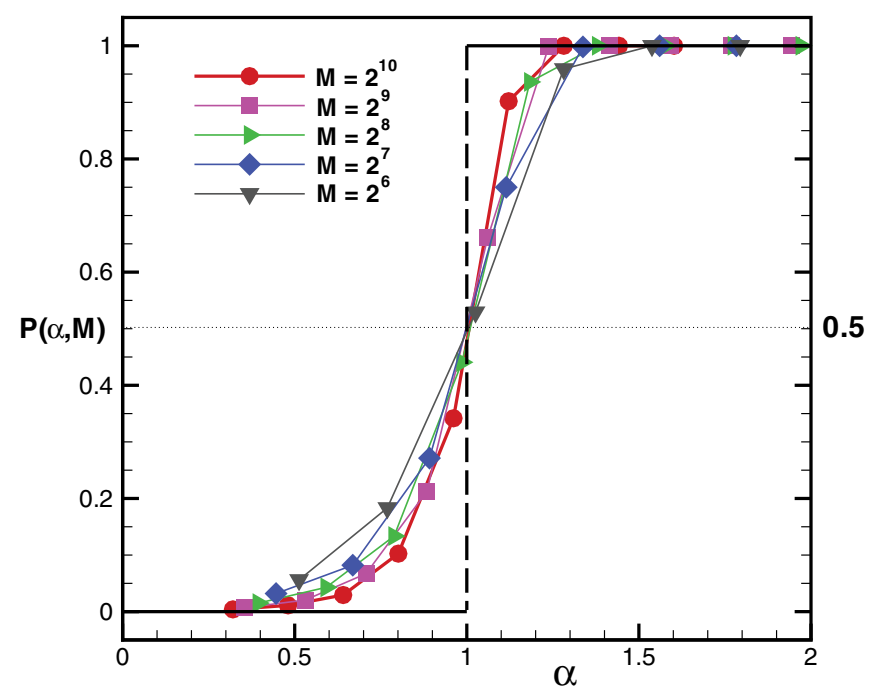

FIG. 3. (Color online) Winning probability $P(\alpha, M)$ curve as a function of the intensive control parameter $\alpha=k / k_{c}(M)$, where $k_{c}(M)=a \ln (M)+b$ is a scaling function of the crossover value with size (the fitting values are $a=0.84$ and $b=0.39$ ) according to Fig. 2, for increasing system sizes. The curves get sharper as $M$ increases, indicating a phase transition in the thermodynamic limit: the onset of the so-called threshold phenomenon. The Heaviside function, reached only in the thermodynamic limit, is a result of the theory (see the text).

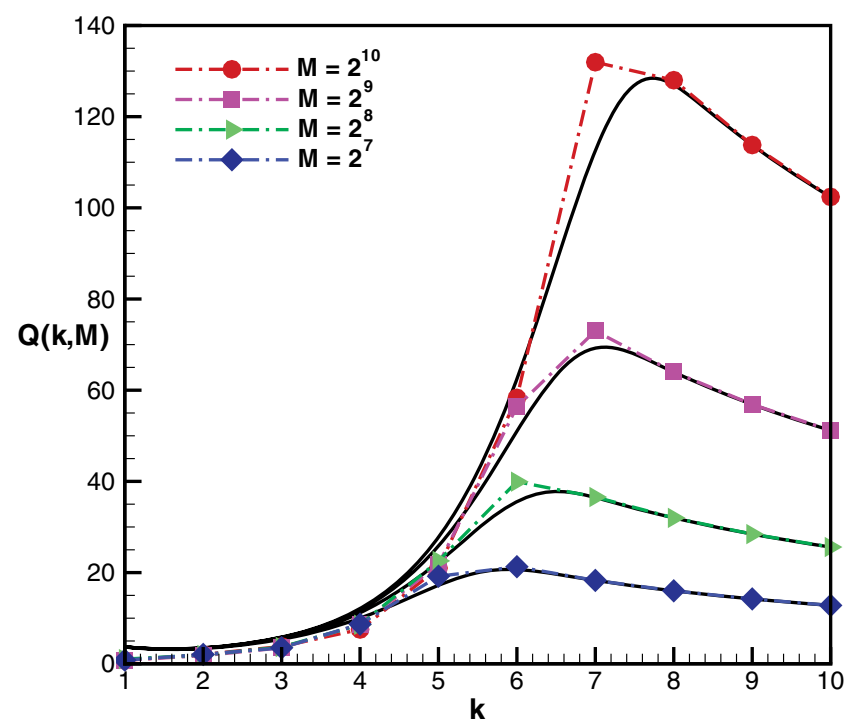

FIG. 4. (Color online) Efficiency measure $Q(k, M)$ curve as a function of $k$, for increasing pool sizes $M$, in the system with $\mathcal{A}=$ $\{+,-, \times, \div\}$. Solid curves are the analytical predictions [Eq. (6)]. This measure is maximized near the transition point.

thermodynamic limit it diverges for $\alpha \rightarrow 1$, deepending on the conjecture that states that the complexity of multicomponent systems is maximized close to their critical points [11,12].

This behavior is also related to the so-called easy-hard-easy pattern that takes place in algorithmic phase transitions and suggests that close to the critical point (hard phase) the computation time that the algorithm needs to come up with a solution is maximized: For $\alpha<1$ the algorithm easily finds that the problem is unsatisfiable, whereas for $\alpha>1$ the algorithm easily finds one of many solutions; when $\alpha \sim 1$ the number of solutions per unit number is optimal and the algorithm needs to perform an exhaustive search of the whole space to find it. Note that within the quiz show Countdown, the pool is bounded to $M=1000$. Interestingly enough, the contestants are allowed to make use of $k=6$ numbers, which corresponds, according to our previous analysis, to the threshold between almost surely unsolvable to almost surely solvable instances. Driving the system towards the critical point ensures that the game is hard but typically solvable, that is, interesting.

To summarize, in this work we have presented a random combinatorial decision problem that evidences an algorithmic phase transition separating the parameter space where the problem is either almost surely satisfiable or unsatisfiable. This work depends on the relationship between number theory and theoretical physics, whose interface has been shown to potentially promote [13] new approaches in both areas.

Several remarks can be made. Note that, while in physical phase transitions the finite-size scaling is usually in the form of a power law shape $k_{c}(M) \sim M^{a}$ with some finite size exponent $a$, in this problem we find logarithmic scaling $k_{c}(M)=$ $a \ln (M)+b$ (insets of Figs. 1 and 2). From a thermodynamic viewpoint, the logarithmic scaling is not problematic since it lacks an a priori physical (i.e., thermodynamic) interpretation. If the reader is uneasy, we emphasize that in order to 
build a thermodynamic formalism we could always define an alternative control parameter $\tilde{k} \equiv \exp (k)$ in order to recover the usual power law scaling with system size $\tilde{k}_{c}(M) \sim M^{a}$ and make this parameter intensive (a temperature) through $\tilde{\alpha}=\tilde{k} / M^{a}$. In contrast, we note that logarithmic scalings have been found previously in other number-theoretic systems evidencing collective phenomena [14-16].

Finally, while the transition between unsatisfiable and satisfiable phases (losing and winning) occurs at lower values in the general case $\mathcal{A}=\{+,-, \times, \div\}$ than in the simple one $\mathcal{A}=\{+,-\}$, in both situations the system evidences the threshold phenomenon. Is this phenomenon restricted to only elementary arithmetic systems or, to the contrary, is this a fundamental behavior in abstract algebraic structures defined as a set of elements with some binary operations?
In this respect, note that the logarithmic scaling $k_{c}(M)$ can be interpreted as the growth rate of the minimal number of elements needed to cover a growing system through binary operations since the number of possible outcomes of combined binary operations grows exponentially fast. This is a challenging open question for future research, which could be addressed within combinatorial group theory [17].

We thank Rémi Monasson for pointing out to us the relation of this problem to combinatorial group theory. We acknowledge financial support from the program MODELICO and Comunidad de Madrid through Grant No. FIS2009-13690. L.L. thanks the Systems and Signals Group at the University of Oxford, where part of this research was developed. The comments of two anonymous referees are also acknowledged.
[1] S. Kirkpatrick and B. Selman, Science 264, 1297 (1994).

[2] R. Monasson, R. Zecchina, S. Kirkpatrick, B. Selman, and L. Troyansky, Nature (London) 400, 133 (1999).

[3] D. Achlioptas, A. Naor, and Y. Peres, Nature (London) 435, 759 (2005).

[4] C. Moore and S. Mertens, The Nature of Computation (Oxford University Press, Oxford, 2011).

[5] Handbook of Satisfiability, edited by A. Biere, M. Heule, H. van Maaren, and T. Walsch (IOS, Amsterdam, 2009).

[6] M. Mezard, G. Parisi, and M. Virasoro, Spin Glass Theory and Beyond (World Scientific, Singapore, 1987).

[7] S. Mertens, Theor. Comput. Sci. 265, 79 (2001).

[8] Wikipedia entry for Countdown game show: http://en.wikipedia.org/wiki/Countdown_(game_show).

[9] L. Lacasa, and B. Luque (unpublished).

[10] G. Hutton, J. Funct. Program. 12, 6 (2002).
[11] J. P. Crutchfield and K. Young, in Complexity, Entropy, and the Physics of Information, edited by W. H. Zurek (Addison-Wesley, Reading, MA, 1990).

[12] R. V. Solé, S. C. Manrubia, B. Luque, J. Delgado, and J. Bascompte, Complexity 2, 49 (1996).

[13] Number theory and physics repository: http:// empslocal.ex.ac.uk/people/staff/mrwatkin/zeta/physics.htm.

[14] B. Luque, L. Lacasa, and O. Miramontes, Phys. Rev. E 76, 010103(R) (2007).

[15] L. Lacasa, B. Luque, and O. Miramontes, New J. Phys. 10, 023009 (2008).

[16] B. Luque, O. Miramontes, and L. Lacasa, Phys. Rev. Lett. 101, 158702 (2008).

[17] M. Gromov, in Geometric Group Theory, edited by G. Niblo and M. Roller (Cambridge University Press, Cambridge, 1993). 